\title{
Casimir Forces between a Gold Sphere and a Thin Graphite Layer on Substrates
}

\author{
Norio Inui* \\ Graduate School of Engineering, University of Hyogo, \\ Shosha 2167, Himeji-shi, Hyogo 671-2280, Japan \\ (Received 12 April 2010; Accepted 6 May 2010; Published 5 June 2010)
}

\begin{abstract}
The Casimir forces between a gold sphere and a thin graphite layer whose thickness is less than $100 \mathrm{~nm}$ are calculated for three types of substrates: gold, silicon, and vitreous silicon oxide. For a fixed separation distance between the sphere and the surface of the graphite layer, the absolute values of the Casimir force increase as the thickness of the graphite layer increases for the substrates of silicon and vitreous silicon oxide, in contrast, the absolute value of the Casimir force decreases for the gold substrate. These changes are pronounced for a small separation distance, particularly for vitreous silicon oxide. Numerical results suggest that the dependence of the Casimir force on the thickness of a graphite layer can be measured using existing technology if the separation distance is sufficiently small. [DOI: 10.1380/ejssnt.2010.287]
\end{abstract}

Keywords: Graphite; Quantum effects; Silicon; Atomic force microscopy

\section{INTRODUCTION}

Carbon materials such as fullerene, carbon nanotubes, and graphene are becoming increasingly important in nanotechnology. In particular, graphene [1] has many unique electrical [2] and mechanical properties [3], and numerous potential applications for graphene have been proposed. Surface reforming by means of graphene is a key application, as surface properties can be drastically modified by covering the surface with graphite layers. For instance, electron mobility is increased by depositing graphite layers on an insulator plate, thus resulting in stretchable transparent electrodes [4]. In addition, the deposition of graphite layers on substrates leads to changes in optical properties [5], which results in the modification of surface forces acting on the substrate.

Although graphite is a familiar material, little is known about surface forces [6-8] acting on graphite layers. In particular, few studies have been conducted on the Casimir force acting on graphite layers, a force that is often referred to as the retarded van der Waals force. Because graphite is a typical anisotropic material, its optical properties have not been studied sufficiently as compared with isotropic materials. The Casimir force acting on graphite layers has not been measured; however, it merits examination. Graphite has several unique properties, the first being its extremely flat surface. The Casimir force between two bodies strongly depends on their separation distance. Thus, the flatness of a specimen is an essential factor in determining the accuracy of the Casimir force. The second distinguishing feature is that we can change the thickness of graphite discretely. Accordingly, we can discuss the dependence of the Casimir force on the thickness of graphite through the number of layers. Here, the natural question is whether a change in the Casimir force acting on a thin graphite layer can be understood by present theories based on assumptions of continuity.

The thinnest graphite layer is graphene. Thus, if there is an anomaly in the dependence of the Casimir force on the thickness of graphite layers that cannot be explained

*Corresponding author: inui@eng.u-hyogo.ac.jp by continuous models, it will be observed most clearly by replacing a single graphene layer with a double graphene layer. An attempt to calculate the Casimir force between a gold plate and suspended graphene was first made by Bordag et al. [9]. using a plasma sheet model. In this model, the graphene layer is described as a negatively charged fluid confined to a plane. Later, a more realistic model based on the Dirac model was proposed by Bordag et al. [10]. However, this model includes an indeterminable parameter; therefore it is at present difficult to predict precisely the Casimir force acting on a graphene sheet theoretically.

Even though graphene has many unknown properties, a suspended graphene sheet is a fascinating candidate for measuring changes in the Casimir force because of the simplicity of the analysis. However, it is not easy to form or handle a large suspended graphene sheet. In addition, the optical properties of suspended graphene may be different from those of graphene on a substrate. Thus, as an initial attempt, it would be better to measure the Casimir force for various thicknesses of multilayer graphene fixed on a variety of substrates. For this reason, in this study, we examine the dependence of the Casimir force on the thickness of graphite fixed on a substrate, using the Lifshitz theory [11], which is based on assumptions of continuity. In particular, we focus on the possibility of measuring changes in the Casimir force acting on a thin graphite layer by existing atomic force microscopy (AFM).

\section{METHOD OF CALCULATION}

Surface forces originate from many interactions such as the Coulomb force, atomic forces, and the Casimir force [12]. If surfaces are not charged with electricity and the separation distance between the surfaces is large, the Casimir force is dominant in these components. The Casimir force results from the change in the zero-point energy of an electromagnetic field. The magnitude of the Casimir force between perfectly conductive parallel plates with a separation distance $a$, which is derived by Casimir [6], is given by

$$
F_{p p}(a)=-\frac{\pi^{2} \hbar c}{240 a^{4}}
$$




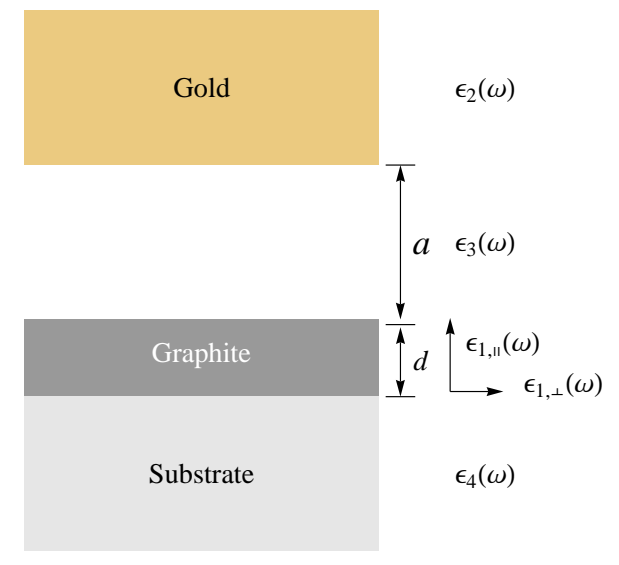

FIG. 1: Configuration of dielectric layers. The graphite layer with thickness $d$ is located on a substrate with infinite thickness. The separation distance between the gold plate and the top surface of the graphite layer is denoted by $a$.

where $\hbar$ is the reduced Planck constant, and $c$ is the speed of light. The Casimir force between two bodies also strongly depends on their shape. Using the proximityforce approximation [8], the Casimir force between a sphere and a plate $F_{s p}$ can be generally expressed by the Casimir energy $E_{p p}(a)$ between two parallel plates as

$$
F_{s p}(a)=2 \pi R E_{p p}(a)
$$

where $R$ is a radius of the sphere, and $a$ is a separation distance that is defined as the minimum distance between the surface of the graphite layer and the sphere. This approximation is valid only for $a \ll R$.

It is extremely difficult to arrange two plates in parallel precisely. Thus, the combination of a sphere and a plate is often used to measure the Casimir force. Mohideen and Roy succeeded in measuring the Casimir force between a sphere with diameter $200 \mu \mathrm{m}$ coated $\mathrm{Au}$ and Pt and a metallic plate [13]. We assume that the Casimir force between a gold sphere and a graphite layer can also be measured by similar methods. The Casimir force between a sphere and a plate is given by eq. (2). Hence, we consider the Casimir energy between a gold plate and a graphite layer on a substrate. The configuration of these objects is shown in Fig. 1.

Each layer shown in Fig. 1 is labeled by an integer $j$, and the Casimir energy at absolute zero is determined by three factors: the separation distance $a$, the thickness of the graphite layer $d$, and the complex dielectric functions $\epsilon_{j}(\omega)$. We assume that a space between a gold plate and a graphite layer is a vacuum. Thus the dielectric function $\epsilon_{3}(\omega)$ takes 1 independently of frequency $\omega$. The dielectric function $\epsilon_{4}(\omega)$ depends on substrates. We assume that the c-axis of the graphite layer is perpendicular to the surface of the substrate. Since graphite has a layered structure, the dielectric function is characterized by $\epsilon_{1, \|}(\omega)$ and $\epsilon_{1, \perp}(\omega)$. According to usage, the subscripts of the dielectric function of graphite are determined on the basis of c-axis.

The Casimir energy for the configuration in Fig. 1 is given by

$$
\begin{aligned}
E(a, d)= & \frac{\hbar}{4 \pi^{2}} \int_{0}^{\infty} k_{\perp} d k_{\perp} \int_{0}^{\infty} d \xi\left\{\ln \left[1-r_{\mathrm{TM}}^{(3,2)}\left(i \xi, k_{\perp}\right) R_{\mathrm{TM}}\left(i \xi, k_{\perp}\right) e^{-2 K_{3}\left(i \xi, k_{\perp}\right) a}\right]\right. \\
& \left.+\ln \left[1-r_{\mathrm{TE}}^{(3,2)}\left(i \xi, k_{\perp}\right) R_{\mathrm{TE}}\left(i \xi, k_{\perp}\right) e^{-2 K_{3}\left(i \xi, k_{\perp}\right) a}\right]\right\}
\end{aligned}
$$

where $k_{\perp}$ is the magnitude of the wave vector component parallel to the surface, and $\xi$ is the frequency variable along the imaginary axis of the electromagnetic field [10]. Using these variables, the function $K_{j}\left(k_{\perp}, \xi\right)$ is defined by

$$
K_{j}\left(k_{\perp}, \xi\right)=\sqrt{k_{\perp}^{2}+\epsilon_{j}(i \xi) \frac{\xi^{2}}{c^{2}}} .
$$

Here, the dielectric function along the imaginary axis $\epsilon_{j}(i \xi)$ is calculated using the Kramers-Kronig dispersion relation

$$
\epsilon_{j}(i \xi)=1+\frac{2}{\pi} \int_{0}^{\infty} \frac{x \epsilon_{j}^{\prime \prime}(x)}{x^{2}+\xi^{2}} d x
$$

where $\epsilon_{j}^{\prime \prime}(\omega)$ denotes the imaginary part of the dielectric function $\epsilon_{j}(\omega)$.

The dependence of the Casimir energy on properties of the materials is determined by the reflection coefficients $r_{\mu}^{(3,2)}$ and $R_{\mu}$, where $\mu \in\{\mathrm{TM}, \mathrm{TE}\}$ denotes the type of mode of electromagnetic wave. The reflection coefficients on the surface between a vacuum and a gold plate along the imaginary axis are given by

$$
\begin{aligned}
r_{\mathrm{TM}}^{(3,2)}\left(i \xi, k_{\perp}\right) & =\frac{\epsilon_{2}(i \xi) K_{3}\left(i \xi, k_{\perp}\right)-\epsilon_{3}(i \xi) K_{2}\left(i \xi, k_{\perp}\right)}{\epsilon_{2}(i \xi) K_{3}\left(i \xi, k_{\perp}\right)+\epsilon_{3}(i \xi) K_{2}\left(i \xi, k_{\perp}\right)} \\
r_{\mathrm{TE}}^{(3,2)}\left(i \xi, k_{\perp}\right) & =\frac{K_{3}\left(i \xi, k_{\perp}\right)-K_{2}\left(i \xi, k_{\perp}\right)}{K_{3}\left(i \xi, k_{\perp}\right)+K_{2}\left(i \xi, k_{\perp}\right)}
\end{aligned}
$$


Let us consider the reflection coefficients for a graphite layer. Graphite is a semimetal with a uniaxial layered crystal structure. Thus, the dielectric function of a graphite layer is characterized by $\epsilon_{1, \|}(\omega)$ and $\epsilon_{1, \perp}(\omega)$, which correspond to the dielectric function of electric-field vector parallels and perpendicular to the c-axis, respectively. Then the reflection coefficients on the surface between a vacuum and a graphite layer along the imaginary axis are given by

$$
\begin{aligned}
r_{\mathrm{TM}}^{(3,1)}\left(i \xi, k_{\perp}\right) & =\frac{\epsilon_{1}(i \xi) K_{3}\left(i \xi, k_{\perp}\right)-\epsilon_{3}(i \xi) K_{1, \|}\left(i \xi, k_{\perp}\right)}{\epsilon_{1}(i \xi) K_{3}\left(i \xi, k_{\perp}\right)+\epsilon_{3}(i \xi) K_{1, \|}\left(i \xi, k_{\perp}\right)}, \\
r_{\mathrm{TM}}^{(1,4)}\left(i \xi, k_{\perp}\right) & =\frac{\epsilon_{4}(i \xi) K_{1, \|}\left(i \xi, k_{\perp}\right)-\epsilon_{1}(i \xi) K_{4}\left(i \xi, k_{\perp}\right)}{\epsilon_{4}(i \xi) K_{1, \|}\left(i \xi, k_{\perp}\right)+\epsilon_{1}(i \xi) K_{4}\left(i \xi, k_{\perp}\right)}, \\
r_{\mathrm{TE}}^{(3,1)}\left(i \xi, k_{\perp}\right) & =\frac{K_{3}\left(i \xi, k_{\perp}\right)-K_{1, \perp}\left(i \xi, k_{\perp}\right)}{K_{3}\left(i \xi, k_{\perp}\right)+K_{1, \perp}\left(i \xi, k_{\perp}\right)}, \\
r_{\mathrm{TE}}^{(1,4)}\left(i \xi, k_{\perp}\right) & =\frac{K_{1, \perp}\left(i \xi, k_{\perp}\right)-K_{4}\left(i \xi, k_{\perp}\right)}{K_{1, \perp}\left(i \xi, k_{\perp}\right)+K_{4}\left(i \xi, k_{\perp}\right)},
\end{aligned}
$$

where $\epsilon_{1}=\sqrt{\epsilon_{1, \|} \epsilon_{1, \perp}}$, and $K_{\|, 1}$ and $K_{\perp, 1}$ are defined as

$$
\begin{aligned}
K_{1, \|}\left(i \xi, k_{\perp}\right) & =\sqrt{k_{\perp}^{2}+\epsilon_{1, \|}(i \xi) \frac{\xi^{2}}{c^{2}}} \\
K_{1, \perp}\left(i \xi, k_{\perp}\right) & =\sqrt{k_{\perp}^{2}+\epsilon_{1, \perp}(i \xi) \frac{\xi^{2}}{c^{2}}} .
\end{aligned}
$$

Finally, the reflection coefficients on the substrate are expressed as

$$
\begin{aligned}
R_{\mathrm{TM}}\left(i \xi, k_{\perp}\right) & =\frac{r_{\mathrm{TM}}^{(3,1)}+r_{\mathrm{TM}}^{(1,4)} e^{-2 K_{1, \|} d}}{1+r_{\mathrm{TM}}^{(3,1)} r_{\mathrm{TM}}^{(1,4)} e^{-2 K_{1, \|} d}}, \\
R_{\mathrm{TE}}\left(i \xi, k_{\perp}\right) & =\frac{r_{\mathrm{TE}}^{(3,1)}+r_{\mathrm{TE}}^{(1,4)} e^{-2 K_{1, \perp} d}}{1+r_{\mathrm{TE}}^{(3,1)} r_{\mathrm{TE}}^{(1,4)} e^{-2 K_{1, \perp} d}} .
\end{aligned}
$$

Consequently, the Casimir force can be calculated from the optical data for the imaginary part of the dielectric function using the Kramers-Kronig relation.

\section{RESULTS AND DISCUSSION}

We consider the Casimir force between a gold sphere and a graphite layer on three types of substrates: gold, silicon, and vitreous silicon oxide. The dielectric functions of these materials along the imaginary axis required to calculate the Casimir force, are calculated via the optical data obtained by experiments with the help of the Kramers-Kronig relation. Here we note that the imaginary component of the dielectric function of gold diverges at $\omega=0$; optical data is not available for low-frequency region. Thus, we use the Drude model:

$$
\epsilon_{2}^{\prime \prime}(\omega)=1-\frac{\omega_{p}^{2}}{\omega(\omega+i \gamma)},
$$

where $\omega_{p}=7.50 \mathrm{eV}$ and $\gamma=0.061 \mathrm{eV}$ for $\hbar \omega<0.125 \mathrm{eV}$ [14].

Figure 2 shows the dielectric functions along the imaginary axis for gold [15], silicon, [16] and vitreous silicon oxide [17]. The magnitude of the dielectric function of

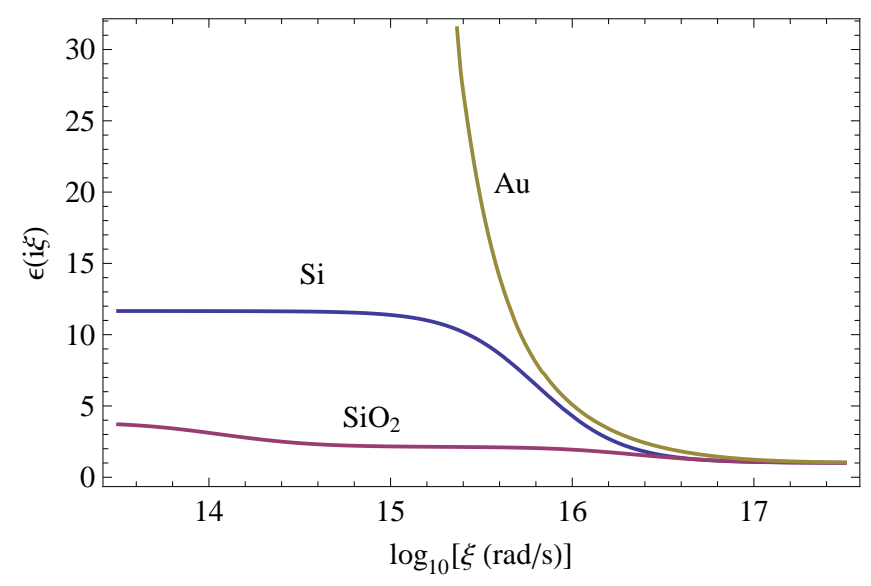

FIG. 2: Dielectric functions along an imaginary frequency of gold, silicon, and vitreous silicon oxide.

gold $\epsilon_{2}^{\prime \prime}(i \xi)$ is much larger than that of other materials in the low-frequency region.

For graphite, Blagov et al. [18] expressed the dielectric functions as

$$
\epsilon_{1, \|}^{\prime \prime}(\omega)=\left\{\begin{array}{l}
\epsilon_{0}^{\prime \prime}=\text { const. } \quad \omega \leq \Omega_{1, \|}, \\
\epsilon_{\|}^{(t)}(\omega), \quad \Omega_{1, \|}<\omega<\Omega_{2, \|} \\
\frac{A_{\|}}{\omega^{3}}, \quad \Omega_{2, \|} \leq \omega
\end{array}\right.
$$

and

$$
\epsilon_{1, \perp}^{\prime \prime}(\omega)=\left\{\begin{array}{l}
\frac{\omega_{p, g}^{2} \gamma_{g}}{\omega\left(\omega^{2}+\gamma_{g}^{2}\right)}, \quad \omega \leq \Omega_{1, \perp} \\
\epsilon_{\perp}^{(t)}(\omega), \quad \Omega_{1, \perp}<\omega<\Omega_{2, \perp} \\
\frac{A_{\perp}}{\omega^{3}}, \quad \Omega_{2, \perp} \leq \omega
\end{array}\right.
$$

where $\epsilon_{\|}^{(t)}(\omega)$ and $\epsilon_{\perp}^{(t)}(\omega)$ are obtained from the tables in Ref. [19]. The imaginary part of the dielectric function $\epsilon_{1, \perp}^{\prime \prime}$ for the low-frequency region is described by Drude model, in which the plasma frequency and the relaxation parameter are determind as $\omega_{p, g}=1.226 \mathrm{eV}$ and $\gamma_{g}=$ $0.04 \mathrm{eV}$. The other parameters in (17) and (18) are determined as $A_{\|}=3.49 \times 10^{4} \mathrm{eV}^{3}$ and $A_{\perp}=9.60 \times 10^{3} \mathrm{eV}^{3}$ 

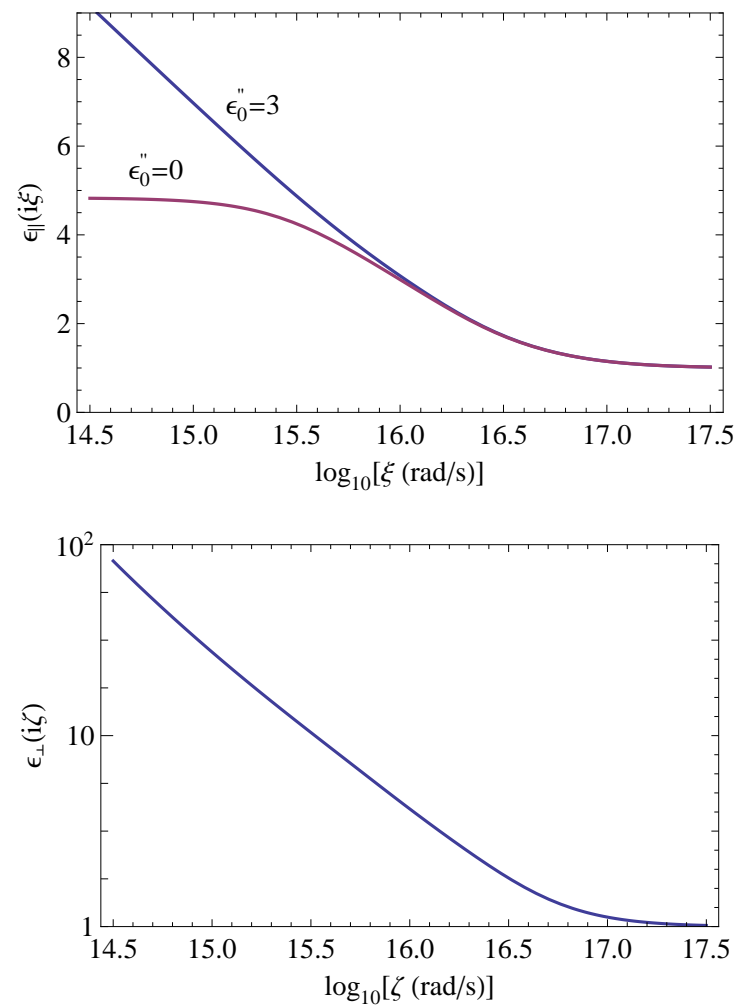

FIG. 3: Dielectric functions of graphite along an imaginary frequency. Two possible dielectric functions with respect to $\epsilon_{1, \perp}(i \xi)$ are shown for $\epsilon_{0}^{\prime \prime}=0$ and $\epsilon_{0}^{\prime \prime}=3$. The component vertical to the surface of graphite $\epsilon_{1, \|}(i \xi)$ diverges in the limit of $\xi \rightarrow 0$.

from the condition of continuity at $\Omega_{2, \|}=\Omega_{2, \perp}=40.0$ $\mathrm{eV}$. As pointed out in Ref. [18], contradictory measurements for $\epsilon_{1, \|}^{\prime \prime}$ are reported for the low-frequency region. Thus, we consider two cases: $\epsilon_{0}^{\prime \prime}=3$ and $\epsilon_{0}^{\prime \prime}=0$. Although Blagov et al. set both $\Omega_{1, \|}$ and $\Omega_{1, \perp}$ to $0.02 \mathrm{eV}$, we set $\Omega_{1, \|}$ to $2 \mathrm{eV}$ because we would like to discuss the possibility of measuring layer, via existing AFM change in the Casimir force by decreasing the thickness of the graphite. Although the deviation of the Casimir force for two cases is enlarged by this modification, as we show later, it is small especially for small $d$. Figure 3 shows dielectric functions along the imaginary axis for graphite. The magnitude of the dielectric functions perpendicular to the graphite surface is much smaller than that parallel to the graphite surface. A large deviation of $\epsilon_{1, \perp}(i \xi)$ is seen for the low-frequency region.

Substituting the obtained dialectic functions along the imaginary frequency into the Lifshitz formula (3), the Casimir energy can be evaluated by numerical integration. Recall that the Casimir force between a sphere and a plate is obtained using the proximity approximation in eq. (2) from the Casimir energy. As a result, the Casimir force between a sphere and a plate with thickness $d$, denoted $F(a, d)$, is given by

$$
F(a, d)=2 \pi R E(a, d) .
$$

Figure 4 shows the dependence of the Casimir force between a gold sphere with a diameter of $200 \mu \mathrm{m}$ and a graphite layer with thickness $d$ on three substrates for
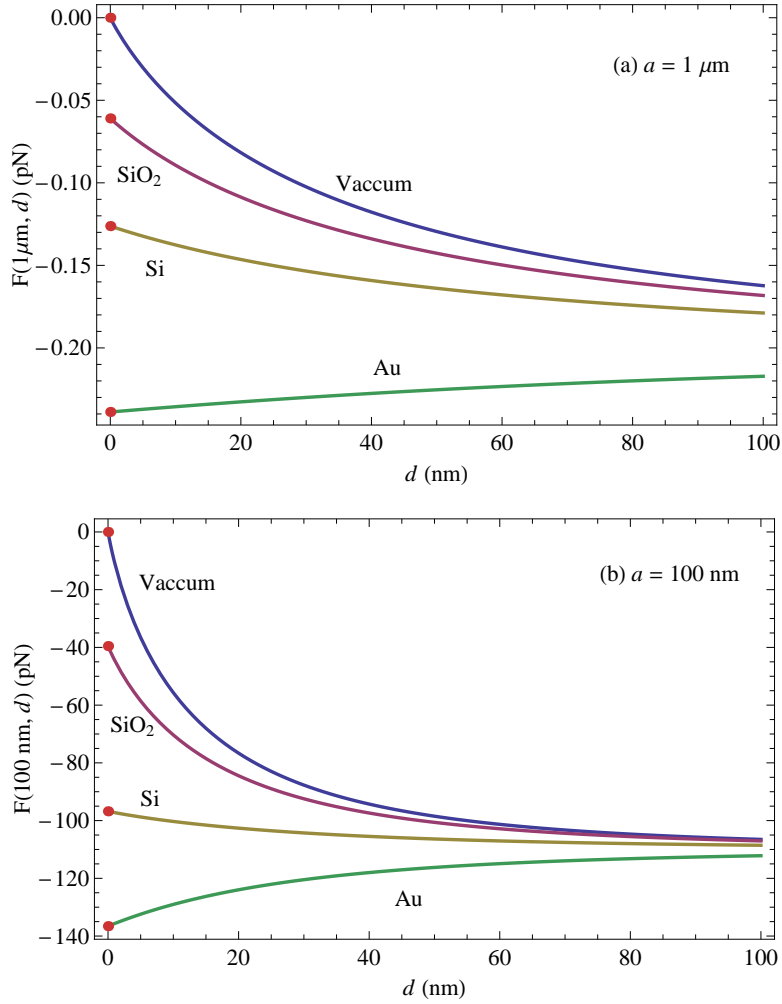

FIG. 4: Plots of the Casimir force between a gold sphere and a graphite layer on three different substrates as a function of the thickness of the graphite layer for separation distance $a=1$ $\mu \mathrm{m}$ and $100 \mathrm{~nm}$. Solid circles denote the Casimir force in the absence of the graphite layers.

two separation distances $1 \mu \mathrm{m}$ and $100 \mathrm{~nm}$. The Casimir force between a gold sphere and suspended graphite is also shown. The solid circles in Fig. 4 indicate the Casimir force in the absence of the graphite layer. We found that the absolute values of the Casimir forces decreases as the thickness of the graphite layer decreases except in the case of the gold substrate. The change in the Casimir force is particularly large where the graphite layer is thin. Furthermore, changes in the Casimir force at the separation $a=100 \mathrm{~nm}$ are much larger than those at $a=1 \mu \mathrm{m}$. From the experimental viewpoint, it is desirable that the change in the Casimir force can be measured distinctly as the thickness decreases. Hence, vitreous silicon oxide is the most suitable substrate of the three materials; moreover, a method for transferring graphene to the substrate of vitreous silicon oxide by using Scotch tape has already been established. The reason that the absolute value of the Casimir force increases as the thickness of the graphite layer decreases only in the case of the gold substrate is that the dielectric function of gold $\epsilon_{2}(i \xi)$, is larger than that of graphite.

In a continuous model, the Casimir force acting on a suspended graphite layer continuously converges to zero as the thickness decreases. However, natural graphite consists of a graphene sheet. Thus, the Casimir force acting on an actual graphite layer must change discretely. Although we are interested in this change, few studies have investigated the Casimir force acting on suspended graphene. Using the Dirac model, Bordag et al. calcu- 

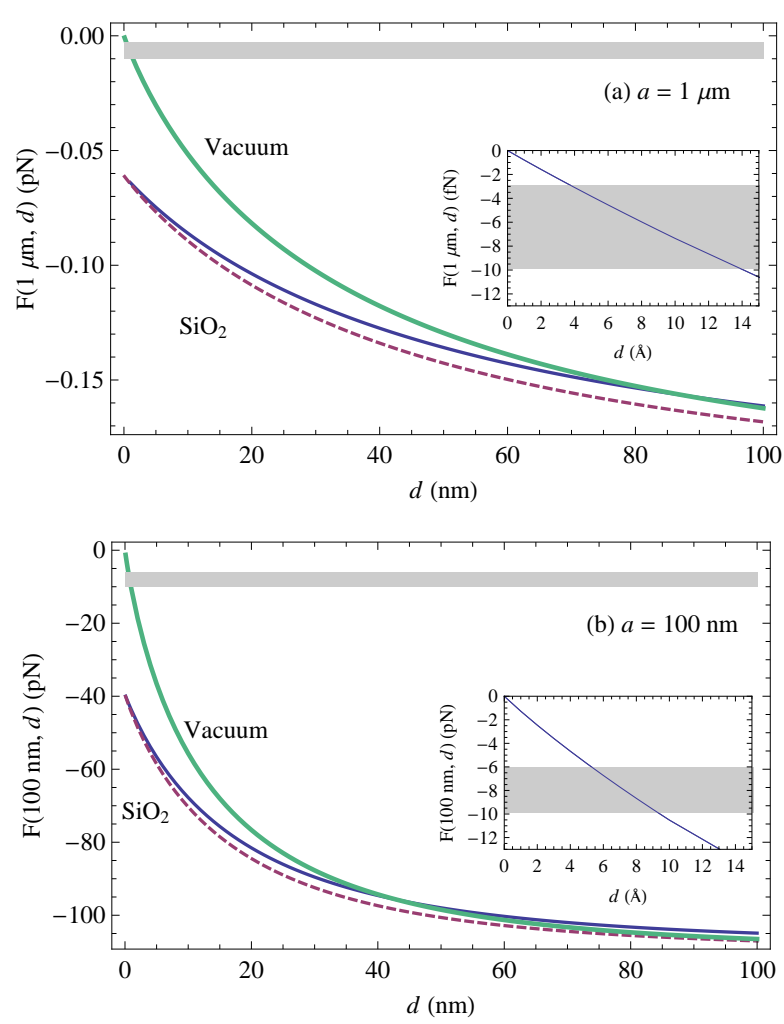

FIG. 5: Plots of deviation resulted from the difference in $\epsilon_{0}^{\prime \prime}$ of $\epsilon_{1, \|}^{\prime \prime}(\omega)$. The Casimir forces between a gold sphere and a graphite layer on vitreous silicon oxide for separation distances for $\epsilon_{0}^{\prime \prime}=0$ and $\epsilon_{0}^{\prime \prime}=3$. The blue solid lines and red dashed lines show the Casimir force using the dielectric functions of graphite corresponding to $\epsilon_{0}^{\prime \prime}=0$ and $\epsilon_{0}^{\prime \prime}=3$, respectively. The green line shows the Casimir force between a gold sphere and suspended graphene. The gray region shows the Casimir force between a perfect conductive sphere with $100 \mu \mathrm{m}$ radius and suspended graphene, calculated using the Dirac model.

lated the Casimir force acting on suspended graphene and a perfectly conductive plate. The gray region in Fig. 5 show the Casimir force between suspended graphene and a perfectly conductive sphere. The reason that the Casimir force is not determined uniquely is that the force depends on a mass gap parameter $m$ (see Ref. [10] for further details). The maximum absolute values and minimum absolute values correspond to $m=0 \mathrm{eV}$ and $m=0.05$ $\mathrm{eV}$, respectively. The actual value of $m$ is probably much smaller than $0.05 \mathrm{eV}$.

The Casimir force acting on suspended graphite in the continuous model increases continuously from zero as the thickness increases. Thus, it becomes equal to the Casimir force acting on a suspended single graphene sheet, obtained by the Dirac model at a certain thickness. We define this thickness as the effective thickness of a single graphene sheet. The effective thickness depends on both the separation distance and the mass gap parameter. If the mass gap parameter is zero, the effective thicknesses are estimated as $14 \AA, 9.3 \AA$ and $9.4 \AA$ for $a=1 \mu \mathrm{m}, 100$ $\mathrm{nm}$, and $40 \mathrm{~nm}$, respectively. If the mass gap parameter is $0.05 \mathrm{eV}$, the effective thicknesses are estimated as 3.7 $\AA$, $5.3 \AA$, and $6.6 \AA$ for $a=1 \mu \mathrm{m}, 100 \mathrm{~nm}$, and $40 \mathrm{~nm}$, respectively.

To consider the dependence of the Casimir force be-

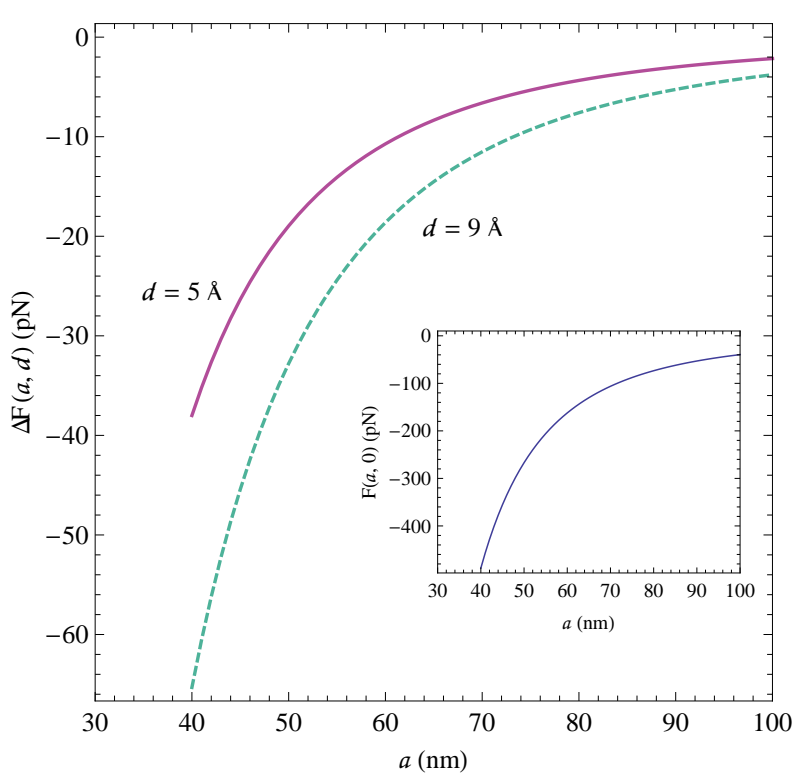

FIG. 6: Plot of the difference in the Casimir force $\Delta F(a, d)$ for $d=5 \AA$ and $d=9 \AA$. The inset shows the Casimir force between a gold sphere and a silica plate.

tween a gold sphere and a graphene layer on vitreous silicon oxide, let us regard a graphene sheet as a graphite layer with thickness $5 \AA$. The solid line in Fig. 6 shows the difference in the Casimir forces, defined by $\Delta F(a, 5 \AA)=$ $F(a, 5 \AA)-F(a, 0)$ for vitreous silicon oxide, and the solid line in the inset shows $F(a, 0)$. If a graphene layer corresponds to a graphite layer with thickness $9 \AA$, the difference in the Casimir forces is shown by the dashed line in Fig. 6.

The difference due to the presence of a graphene layer becomes pronounced as the separation between the plate and sphere decreases. If the resolution of the force of $\mathrm{AFM}$ is $10 \mathrm{pN}$, the presence of a graphene layer can be detected by bringing the sphere toward the plate with separations of less than $50 \mathrm{~nm}$. The difference in Casimir forces at small gaps becomes large; however, the Casimir force itself also becomes strong as shown in the inset in Fig. 6. Thus, the position at which pull-in of the gold sphere to the graphene layer appears will determine the observed maximum difference of the Casimir force.

\section{CONCLUSION}

We have calculated the Casimir force between a gold sphere with $200 \mu \mathrm{m}$ diameter and a graphite layer on three substrates of $\mathrm{Au}, \mathrm{Si}$, and $\mathrm{SiO}_{2}$. When the thickness of the graphite layer was changed, the largest change in the Casimir force was observed for the substrate of vitreous silicon oxide. In particular, the Casimir force changed clearly in the region of small thickness. This raises the expectation of measuring change in the Casimir force when a substrate of silicon oxide is covered by a single graphene layer.

Unfortunately, the optical properties of graphene on vitreous silicon oxide have not yet been measured. Thus, we cannot calculate the Casimir force accurately. We 
therefore regarded a graphite layer with a thickness of 5 $\AA$ as a graphene layer and estimated the Casimir between a gold sphere and graphene on vitreous silicon oxide. Our results suggest that the Casimir force is strengthened by the presence of a graphene layer, and the difference in the Casimir force in the absence of the graphene layer exceeds $10 \mathrm{pN}$ when the separation distance is below $50 \mathrm{~nm}$.

The diameter of the gold sphere used in our calculation is the same as that used in the experiment by Mohideen and Roy [13]. In addition, since AFM with picoNewton resolution is already often used to measure the Casimir force, it should be possible to measure change in the Casimir force, as shown in this study, using the existing technology.

Recently, we studied whether a graphene sheet levitates by the repulsive Casimir force [20]. The necessary condition under which levitation occurs is that the repulsive Casimir force is stronger than the gravity acting on graphene. If the repulsive Casimir force increases as the number of layers of graphene increases, similarly to the change examined in this study, and the increment of the Casimir force by increasing the number of layers exceeds that of gravity, there should be an optimal number of layers for realizing the maximum height of levitation. Although the levitation by the repulsive Casimir force in vacuum has not been realized yet, the repulsive Casimir force between a gold sphere and vitreous silicon oxide separated by bromobenzene has been observed [14] The condition that the dielectric function of bromoben- zene is larger than that of vitreous silicon and smaller than that of gold is important to realize the repulsive Casimir force. Since the value of the dielectric function of $\epsilon_{1, \perp}$ is much larger than that of vitreous silicon. Thus, the Casimir force between graphite and vitreous silicon oxide separated by bromobenzene may be repulsive.

Regarding potential application, we may be able to utilize measurement of the Casimir force between a gold sphere and a multi-layered graphene layer to determine the number of layers. Optical determination of the thickness is the most popular method [21] and is based on the fact that the reflection coefficients of the multi-layered graphene depend on the number of layers of graphene. The change in the Casimir force also originates in the change in the reflection coefficients. However, the advantage of this method is that measurement of the topography and the number of layers can be achieved simultaneously using only one AFM procedure. In contrast to ordinary AFM, the Casimir force microscope may detect a void inside a graphite layer if the void is sufficiently large.

\section{Acknowledgments}

The author would like to Kouji Miura and Makoto Ishikawa for helpful discussions.
[1] K. S. Novoselov, A. K. Geim, S. V. Morozov, D. Jiang, Y. Zhang, S. V. Dubonos, I. V. Grigorieva, and A. A. Firsov, Science 306, 666 (2004).

[2] K. S. Novoselov, A. K. Geim, S. V. Morozov, D. Jiang, M. I. Katsnelson, I. V. Grigorieva, S. V. Dubonos, and A. A. Firsov, Nature 438, 197 (2005).

[3] C. Lee, X. Wei, J. W. Kysar, and J. Hone, Science 321, 385 (2008).

[4] K. S. Kim, Y. Zhao, H. Jang, S. Y. Lee, J. M. Kim, K. S. Kim, J-H. Ahn, P. Kim, J-Y Choi, and B. H. Hong, Nature 457, 706 (2009).

[5] L. A. Falkovsky and S. S. Pershoguba, Phys. Rev. B 76, 153410 (2007).

[6] H. B. G. Casimir, Proc. Kon. Ned. Akad. Wet. 51, 793 (1948).

[7] P. W. Mionni, The Quantum Vacuum (Academic Press, SanDiego 1994).

[8] M. Bordag, B. Geyer, G. L. Klimchitskaya, U. Mohideen, and V. M. Mostepanenko, Advances in the Casimir Effect (Oxford University Press, New York 2009).

[9] M. Bordag, B. Geyer, G. L. Klimchitskaya, and V. M. Mostepanenko, Phys. Rev. Rev. B 74, 205431 (2006).

[10] M. Bordag, I. V. Fialkovsky, D. M. Gitman, and D. V. Vassilevich, Phys. Rev. B 80, 245406 (2009).

[11] L. D. Landau and E. M. Lifshitz, Electrodynamics of Continuous Media (Oxford Science Publications, SanDiego, 1982).
[12] J. N. Israelachvili Intermolecular and Surface Forces (Academic Press, London, 1992).

[13] U. Mohideen and A. Roy, Phys. Rev. Lett. 81, 4549 (1998).

[14] J. N. Munday, F. Cappasso, and V. A. Parsegian, Nature 457, 170 (2009).

[15] D. W. Lynch and W. R. Hunter, in: Gold(Au) Handbook of Optical Constants of Solids, ed. E. D. Palik (Academic, New York, 1991) p. 286.

[16] D. F. Edwards, in: Silicon(Si) Handbook of Optical Constants of Solids, ed. E. D. Palik (Academic, New York, 1991) p. 547.

[17] H. R. Philipp, in: Silicon Dioxide $\left(\mathrm{SiO}_{2}\right)$ (Glass) Handbook of Optical Constants of Solids, ed. E. D. Palik (Academic, New York, 1991) p. 749.

[18] E. V. Blagov, G. L. Klimchitskaya, and V. M. Mostepanenko, Phys. Rev. B 71, 235401 (2005).

[19] A. Borghesi and G. Guizzetti, in: Graphite(C), Handbook of Optical Constants of Solids, ed. E. D. Palik (Academic, New York, 1991) p. 460.

[20] N. Inui and K. Miura, e-J. Surf. Sci. Nanotech. 8, 57 (2010).

[21] Z. H. Ni, H. M. Wang, J. Kasim, H. M. Fan, T. Yu, Y. H. Wu, Y. P. Feng, and Z. X. Shen, Nano Lett. 7, 2758 (2007). 